\title{
THETA DIVISORS OF STABLE VECTOR BUNDLES MAY BE NONREDUCED
}

\author{
GEORGE H. HITCHING \\ WITH AN APPENDIX BY CHRISTIAN PAULY
}

\begin{abstract}
A generic strictly semistable bundle of degree zero over a curve $X$ has a reducible theta divisor, given by the sum of the theta divisors of the stable summands of the associated graded bundle. The converse is not true: Beauville and Raynaud have each constructed stable bundles with reducible theta divisors. For $X$ of genus $g \geq 5$, we construct stable vector bundles over $X$ of rank $r$ for all $r \geq 5$ with reducible and nonreduced theta divisors. We also adapt the construction to symplectic bundles.

In the appendix, Raynaud's original example of a stable rank 2 vector bundle with reducible theta divisor over a bi-elliptic curve of genus 3 is generalized to bi-elliptic curves of genus $g \geq 3$.
\end{abstract}

\section{INTRODUCTION}

Let $X$ be a complex projective smooth curve of genus $g \geq 2$. We write $J$ for the Jacobian variety parametrizing line bundles of degree $g-1$ over $X$. To a vector bundle $V \rightarrow X$ of degree zero we may associate the set

$$
\left\{N \in J: h^{0}(N \otimes V)>0\right\} .
$$

If $V$ is a generic semistable bundle of rank $n$, then this is the support of a divisor $\Theta_{V}$ on $J$, called the theta divisor of $V$, which is algebraically equivalent to $n \Theta$. See Beauville [2, 3, for details. (For certain nongeneric semistable or stable $V$, then (1.1) is the whole of $J$. This phenomenon was first studied by Raynaud [14], and has subsequently attracted a good deal of attention.)

Laszlo has given an analogue of the Riemann singularity theorem for $\Theta_{V}$ :

Theorem 1.1. If $\Theta_{V}$ is defined, then mult $_{N} \Theta_{V} \geq h^{0}(N \otimes V)$.

Proof. This follows easily from [12, Proposition V.2]. Laszlo's statement is for rank 2 bundles of slope $g-1$, but the arguments are easily adapted to bundles of slope zero, and apply in arbitrary rank if one assumes that $\Theta_{V}$ is defined.

If $V$ is strictly semistable, S-equivalent to a decomposable bundle $\bigoplus_{i} V_{i}$ where each $V_{i}$ is stable of degree zero, then $\Theta_{V}$ has the reducible theta divisor $\sum_{i} \Theta_{V_{i}}$, when this exists. The converse is not true; the first counterexample was given by Raynaud, who constructed a stable rank two bundle over a bi-elliptic curve of genus 3 with a reducible theta divisor. This work was never published; in the appendix by Christian

2010 Mathematics Subject Classification. 14H60, $14 \mathrm{H} 40$.

Key words and phrases. Vector bundle, curve, theta divisor. 
Pauly to the present article, Raynaud's construction is described and generalized to bi-elliptic curves of genus $g \geq 3$.

In [3], Beauville constructed stable bundles with reducible theta divisors over a general curve $X$ of genus $g \geq 3$, of ranks $\left(\begin{array}{l}g \\ p\end{array}\right)$, for $1 \leq p \leq g-1$. These are of the form $\bigwedge^{p} E_{L}$, where $E_{L}$ is the evaluation bundle defined by the exact sequence

$$
0 \rightarrow E_{L}^{*} \rightarrow O_{X} \otimes H^{0}(L) \rightarrow L \rightarrow 0
$$

where $L$ is a general, very ample line bundle of degree $2 g$. (Note that $E_{L}$ has slope 2, so the theta divisor of $\bigwedge^{p} E_{L}$ belongs to $J^{g-3}$ instead of $J=J^{g-1}$.)

In the present work, we study a related phenomenon. If $V$ is a semistable bundle with theta divisor $\Theta_{V}$, then by Theorem 1.1, the polystable bundle $V^{\oplus n}$ has the nonreduced theta divisor $n \Theta_{V}$. In light of Beauville's and Raynaud's constructions, it seems reasonable to expect that there also exist stable bundles with nonreduced theta divisors. In $\S 3$, we construct stable bundles with reducible and nonreduced theta divisors. Precisely:

Theorem 1.2. Suppose $X$ has genus $g \geq 4$. Let $\Theta_{V}$ be the theta divisor of a generic stable bundle $V$ of rank $n \geq 1$ over $X$. Let $t$ be a positive integer with $2 \leq t<n(g-1)$. Then for any rank $r \geq t n+2$, there exist stable bundles $W$ of degree zero and rank $r$ such that $\Theta_{W}$ exists and contains $(t-1) \Theta_{V}$ as a subscheme.

In particular, letting $t=2$ or 3 and $n=1$, we obtain:

Corollary 1.3. Over any curve of genus $g \geq 5$, there exist stable bundles of all ranks $r \geq 4$ (resp., $r \geq 5$ ) with reducible (resp., reducible and nonreduced) theta divisor.

These $W$ are obtained as extensions $0 \rightarrow E \rightarrow W \rightarrow M \rightarrow 0$ where $E$ is a stable bundle of degree -1 with low Segre invariants and "large" families of maximal subbundles for certain ranks. These $E$, which we construct in $\Upsilon_{2}$, are similar to examples of Ballico and Russo [1] of bundles whose Quot scheme $M_{k}(E)$ of maximal subbundles of rank $k$ is of large dimension.

In \$5, we adapt the construction to produce symplectic bundles $W$ of even rank $\geq 6$ with reducible theta divisors, and nonreduced if $r \geq 8$. These are obtained as extensions $0 \rightarrow E \rightarrow W \rightarrow E^{*} \rightarrow 0$ where $E$ is as above. To perform this construction, we obtain in $\$ 4$ some results on liftings in symplectic extensions which we hope may also be applicable in other contexts.

Acknowledgements: I thank Insong Choe for helpful comments on this work, and Christian Pauly and Michel Raynaud for information on the construction in the appendix.

\section{Stable BUndLes With many MaXimal SUbBUndles}

In this section, we construct the bundles $E$ referred to in the introduction. We begin by recalling some results on vector bundle extensions. 
2.1. Extensions, lifting and geometry. Let $E$ and $F$ be vector bundles over a curve, and let $0 \rightarrow E \rightarrow W \rightarrow F \rightarrow 0$ be a nontrivial extension. In this section we recall some results on liftings of elementary transformations of $F$ to $W$.

Let $V$ be a vector bundle with $h^{1}(V) \neq 0$, and write $\pi$ for the projection $\mathbb{P} V \rightarrow X$. By Serre duality and the projection formula and since $\pi_{*} O_{\mathbb{P} V}(1)=V^{*}$, we have an identification

$$
H^{1}(X, V) \stackrel{\sim}{\rightarrow} H^{0}\left(\mathbb{P} V, \pi^{*} K_{X} \otimes O_{\mathbb{P} V}(1)\right)^{*} .
$$

By standard algebraic geometry, we obtain a map $\mathbb{P} V \rightarrow \mathbb{P} H^{1}(V)$. See [7, §2] for more information and other descriptions of this map.

If $V=\operatorname{Hom}(F, E)=F^{*} \otimes E$ then we may consider the locus $\Delta_{F^{*} \otimes E}$ of rank one tensors, which has dimension $\operatorname{rk} F+\operatorname{rk} E-1$.

Lemma 2.1. Let $E, F$ and $W$ be as above. If an elementary transformation

$$
0 \rightarrow \tilde{F} \rightarrow F \rightarrow \tau \rightarrow 0
$$

with $\operatorname{deg} \tau \leq k$ lifts to a subsheaf of $W$, then the class $\delta(W)$ of the extension belongs to $\operatorname{Sec}^{k}\left(\psi\left(\Delta_{F^{*} \otimes E}\right)\right)$.

Proof. This is proven in [6, Theorem 4.4 (i)]. Note that in [6] there are various assumptions on the degrees and genericity of $E$ and $F$, which need not be satisfied in the present applications. However, the function of these assumptions is to ensure that $\mathbb{P} \operatorname{Hom}(F, E)$ is embedded in $\mathbb{P} H^{1}(\operatorname{Hom}(F, E))$, which we do not require here.

2.2. Construction of stable bundles with many maximal subbundles. Let $X$ be a curve of genus $g \geq 4$. Here we construct the bundles $E$ mentioned in the introduction. Like the bundles with large $M_{k}(E)$ constructed by Ballico and Russo [1], these $E$ will be extensions of a decomposable bundle by a line bundle.

Choose a generic stable bundle $V \rightarrow X$ of degree zero and rank $n \geq 1$, and a positive integer $t$ with

$$
t<n(g-1) .
$$

Let $L \rightarrow X$ be a line bundle of degree -1 , and consider a generic extension $0 \rightarrow L \rightarrow$ $E \rightarrow V \otimes \mathbb{C}^{t} \rightarrow 0$. The following two lemmas form a partial analogue of the Claim in the proof of [1, Theorem 0.0.1]:

Lemma 2.2. Every subbundle of $E$ has negative degree.

Proof. Let $F$ be a proper subbundle of $E$. Then $F$ fits into a diagram

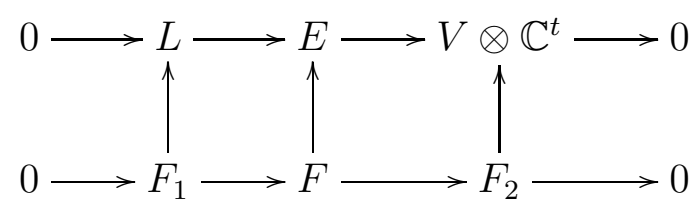

where $F_{1}$ is either zero or $L$, and $F_{2}$ is a subsheaf of $V \otimes \mathbb{C}^{t}$. If $F_{1}=L$ or if $t=1$ then clearly $F$ has negative degree. If $t \geq 2$, we must show that no subbundle of the form $V \otimes \Lambda$ lifts to $E$, where $\Lambda \subset \mathbb{C}^{t}$ is a proper vector subspace. Clearly it suffices to treat the case $\operatorname{dim} \Lambda=1$. We need to check that subspaces of the form

$$
\operatorname{Ker}\left(H^{1}\left(\operatorname{Hom}\left(V \otimes \mathbb{C}^{t}, L\right)\right) \rightarrow H^{1}(\operatorname{Hom}(V \otimes \Lambda, L))\right)
$$


do not sweep out $H^{1}\left(\operatorname{Hom}\left(V \otimes \mathbb{C}^{t}, L\right)\right)$. Since $V$ is stable, $h^{0}(\operatorname{Hom}(V, L))=0$. The dimension of (2.2) is therefore $(t-1) h^{1}(\operatorname{Hom}(V, L))$. Furthermore, the subspaces $\Lambda$ vary in $\mathbb{P}^{t-1}$. Therefore, it suffices to check that

$$
(t-1)+(t-1) h^{1}(\operatorname{Hom}(V, L))<t \cdot h^{1}(\operatorname{Hom}(V, L)),
$$

that is, $t-1<h^{1}(\operatorname{Hom}(V, L))$.

By Riemann-Roch and since $h^{0}(\operatorname{Hom}(V, L))=0$, we have $h^{1}(\operatorname{Hom}(V, L))=n g$. The inequality $t-1<n g$ follows from assumption (2.1), and we are done.

Lemma 2.3. Let $E$ be a generic extension of $V \otimes \mathbb{C}^{t}$ by $L$ as above. Then all degree -1 subbundles of $E$ contain the subbundle $L$.

Proof. We proceed by induction on $t$. It is convenient to begin with the case $t=1$, although in applications we will most often assume that $t \geq 2$.

Consider an extension $0 \rightarrow L \rightarrow E \rightarrow V \rightarrow 0$. Any degree -1 subbundle $F$ of $E$ not containing $L$ must lift from a subsheaf of $V$.

Proposition 2.4. A generic vector bundle $V$ of rank $n \geq 2$ and degree zero over a curve of genus $g \geq 4$ has no subbundles of degree -1 .

Proof. By Russo-Teixidor i Bigas [15, Theorem 0.2], the Quot scheme of subsheaves of degree -1 and rank $m$ of a generic $V$ is empty when the expected dimension $n-m(n-m)(g-1)$ is negative. One checks easily that the maximum value of this dimension occurs at $m=1$ and $m=n-1$, when it is equal to $n-(n-1)(g-1)$. Since $g \geq 4$, the required inequality would follow from $n-3(n-1)<0$, which is clear since $n \geq 2$.

By the proposition, any subbundle $F \subset E$ of degree -1 not containing $L$ must lift from an elementary transformation $F \rightarrow V \rightarrow \mathbb{C}_{x}$. By Theorem 2.1 , this happens only if the extension class of $E$ belongs to the image of the scroll $\mathbb{P H o m}(V, L)$ in $\mathbb{P} H^{1}(\operatorname{Hom}(V, L))$. Since $L$ is a line bundle, $\Delta_{V^{*} \otimes L} \cong \mathbb{P} V^{*}$, which has dimension $n$. On the other hand, $h^{1}(\operatorname{Hom}(V, L))-1=n g-1$. Since $g \geq 4$, a general extension class $\delta(E)$ does not belong to $\Delta_{V^{*} \otimes L}$. Thus we have proven the lemma for $t=1$.

Now suppose $t \geq 2$, and let $E$ be a generic extension $0 \rightarrow L \rightarrow E \rightarrow V \otimes \mathbb{C}^{t} \rightarrow 0$. Choose a subspace $\Lambda \subset \mathbb{C}^{t}$ of dimension $t-1$, and consider the diagram

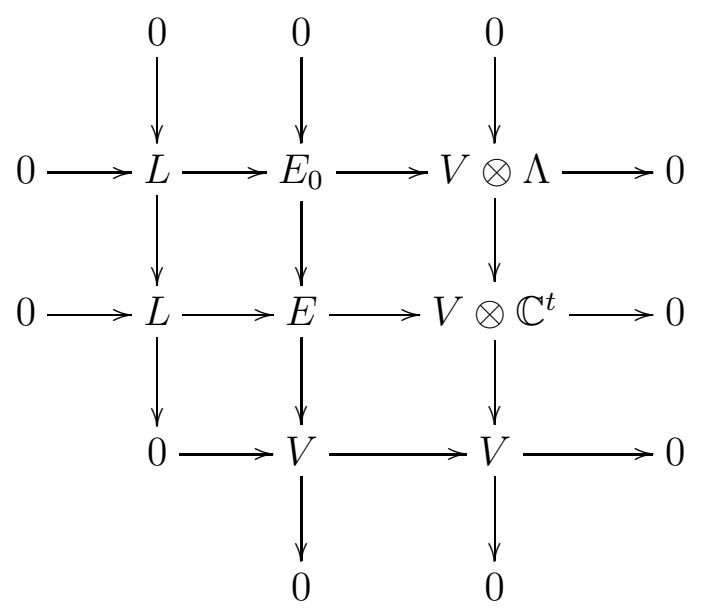


Since the induced map $H^{1}\left(\operatorname{Hom}\left(V \otimes \mathbb{C}^{t}, L\right)\right) \rightarrow H^{1}(\operatorname{Hom}(V \otimes \Lambda, L))$ is surjective, we may assume that $E_{0}$ is a generic extension of $V \otimes \Lambda$ by $L$.

Suppose $F \subset E$ is a proper subbundle of degree -1 . Then we have a diagram

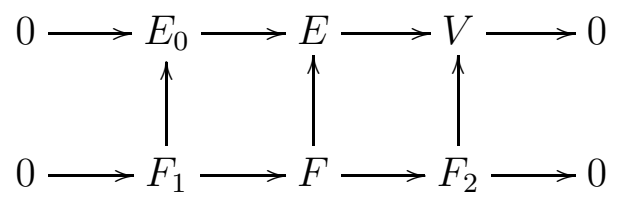

where $F_{1}$ is a subbundle of $E_{0}$ and $F_{2}$ a subsheaf of $V$.

Firstly, suppose $F_{1} \neq 0$. By Lemma 2.2 we have $\operatorname{deg} F_{1} \leq-1$, and therefore $\operatorname{deg} F_{2} \geq 0$. Since $V$ is stable, the only possibilities are $F_{2}=0$ and $F_{2}=V$, and so in fact $\operatorname{deg} F_{1}=-1$. By induction, $L$ belongs to $F_{1}$ and hence to $F$.

On the other hand, if $F_{1}=0$ then $F \cong F_{2}$ must lift from a degree -1 subsheaf of $V$. By Proposition 2.4, the only possibility is that $F_{2}$ is an elementary transformation

$$
0 \rightarrow F \rightarrow V \rightarrow \tau \rightarrow 0
$$

where $\tau$ is a torsion sheaf of degree 1. By Theorem 2.1, the lifting of such an $F$ implies that the class $\varepsilon$ of the extension

$$
0 \rightarrow E_{0} \rightarrow E \rightarrow V \rightarrow 0
$$

belongs to the image of the scroll $\mathbb{P H o m}\left(V, E_{0}\right)$ in $\mathbb{P} H^{1}\left(\operatorname{Hom}\left(V, E_{0}\right)\right)$.

We note that since $E / L$ is a direct sum $V \otimes \mathbb{C}^{t}$, the class $\varepsilon$ belongs to

$$
\operatorname{Ker}\left(H^{1}\left(\operatorname{Hom}\left(V, E_{0}\right)\right) \rightarrow H^{1}(\operatorname{Hom}(V, V \otimes \Lambda))\right),
$$

which has dimension

$$
h^{1}(\operatorname{Hom}(V, L))-h^{0}(\operatorname{Hom}(V, V \otimes \Lambda))=n g-(t-1) .
$$

Conversely, it is easy to see that any element of this kernel gives an extension $E$ of the form we began with.

We claim now that the intersection of $\psi\left(\Delta_{V^{*} \otimes E_{0}}\right)$ with

$$
\operatorname{Im}\left(\mathbb{P} H^{1}(\operatorname{Hom}(V, L)) \rightarrow \mathbb{P} H^{1}\left(\operatorname{Hom}\left(V, E_{0}\right)\right)\right)
$$

is exactly $\psi(\mathbb{P H o m}(V, L))$. Suppose $\psi\left(v^{*} \otimes e_{0}\right) \in \mathbb{P} H^{1}(\operatorname{Hom}(V, L))$ where $e_{0} \notin L$. Write $v_{0}$ for the image of $e_{0}$ in $V \otimes \Lambda$. Then the corresponding point $v^{*} \otimes v_{0}$ is a base point of the natural map

$$
\mathbb{P}(\operatorname{End}(V) \otimes \Lambda) \rightarrow \mathbb{P} H^{1}(\operatorname{End}(V) \otimes \Lambda) .
$$

But it follows from Hwang-Ramanan [10, Proposition 3.2] that this map is base point free (in fact an embedding) for general $V$. Thus if $\psi\left(v^{*} \otimes e_{0}\right) \in \mathbb{P} H^{1}(\operatorname{Hom}(V, L))$ then in fact $e_{0} \in L$.

By the claim, we must check that

$$
\operatorname{dim} \mathbb{P H o m}(V, L)<h^{1}(\operatorname{Hom}(V, L))-h^{0}(\operatorname{Hom}(V, V \otimes \Lambda))-1,
$$

that is, $n<n g-(t-1)-1$. This is exactly the assumption (2.1). Hence a generic extension $0 \rightarrow E_{0} \rightarrow E \rightarrow V \rightarrow 0$ of our preferred type admits no lifting of the form (2.3), and we are done. 
Corollary 2.5. (1) Any subbundle $F \subseteq E$ of degree -1 is of the form $0 \rightarrow L \rightarrow$ $F \rightarrow V \otimes \Lambda \rightarrow 0$, where $\Lambda$ is a uniquely determined vector subspace of $\mathbb{C}^{t}$.

(2) The degree -1 subbundles of $E$ are parametrized by the union of the Grassmann varieties $\operatorname{Gr}\left(\mathbb{C}^{t}, s\right)$ for $s \in\{0, \ldots, t\}$.

Proof. This is straightforward to check, in view of Lemma 2.3 and since $V$ is stable.

\section{Stable BundLES With REDUCible AND NONREDUCED THETA DiVISORS}

We continue to assume that $X$ has genus $g \geq 4$.

Proposition 3.1. Let $M$ be a generic stable bundle of rank $m \geq 1$ and degree 1 . Then any proper subbundle of $M$ has negative degree.

Proof. Similar to Proposition 2.4.

Theorem 3.2. A generic extension $0 \rightarrow E \rightarrow W \rightarrow M \rightarrow 0$ is a stable vector bundle.

Proof. Suppose $G \subset W$ is a proper subbundle. If $G$ is contained in the subbundle $E$ then $\operatorname{deg} G \leq-1$ by Lemma 2.2. Otherwise, we have a diagram

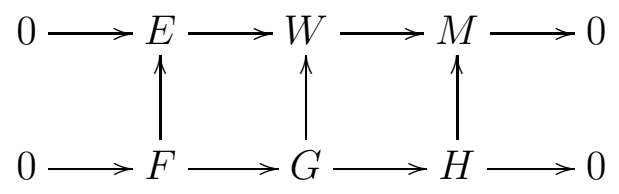

where $F$ is a subbundle of $E$ and $H$ a subsheaf of $M$. By Proposition 3.1, it suffices to exclude liftings of the following types to $W$ :

(i) extensions $0 \rightarrow F \rightarrow G \rightarrow M \rightarrow 0$ where $F \subset E$ has degree -1 ; and

(ii) degree zero elementary transformations of $M$.

(i) Suppose $F \subset E$ is a proper subbundle of degree -1 . By Corollary 2.5 (1), we have $\operatorname{rk} F=s n+1$ for some $0 \leq s<t$. Then an extension $G$ of $M$ by $F$ belongs to $W$ if and only if $\delta(W)$ belongs to

$$
\operatorname{Im}\left(H^{1}(\operatorname{Hom}(M, F)) \rightarrow H^{1}(\operatorname{Hom}(M, E))\right) .
$$

By Corollary 2.5 (2), to exclude case (i) in general, it will suffice to show that

$$
h^{1}(\operatorname{Hom}(M, E))-h^{1}(\operatorname{Hom}(M, F))-\operatorname{dim} \operatorname{Gr}\left(\mathbb{C}^{t}, s\right)>0 .
$$

A straightforward calculation using Riemann-Roch shows that this would follow from $((m(g-1)+1) n-s)(t-s)>0$. Since $t>s$, we have $s<t<n(g-1)$ by (2.1) , and then clearly $((m(g-1)+1) n-s)(t-s)>0$ as desired.

(ii) By Theorem 2.1, a degree 0 elementary transformation of $M$ lifts to $W$ only if the extension class of $W$ belongs to $\psi\left(\Delta_{M^{*} \otimes E}\right)$ in $\mathbb{P} H^{1}(\operatorname{Hom}(M, E))$. We have

$$
\begin{aligned}
\operatorname{dim} \psi\left(\Delta_{M^{*} \otimes E}\right) \leq m & +t n \\
& <m+t n+m(t n+1)(g-1)=\operatorname{dim} \mathbb{P} H^{1}(\operatorname{Hom}(M, E)),
\end{aligned}
$$

so a general extension $W$ admits no such lifting. 
Now we study theta divisors of such extensions $W$. Suppose $t \geq 2$, and let $V, L$, $E$ and $M$ be as above. Since $V$ is generic, we may assume $V$ has a reduced theta divisor $\Theta_{V}$.

Theorem 3.3. A generic extension $0 \rightarrow E \rightarrow W \rightarrow M \rightarrow 0$ has a reducible theta divisor $\Theta_{W}$ which scheme-theoretically contains $(t-1) \Theta_{V}$. In particular, if $t \geq 3$ then $\Theta_{W}$ is also nonreduced.

Proof. For $P \in \Theta_{V}$, consider the exact sequence

$$
0 \rightarrow P \otimes L \rightarrow P \otimes E \rightarrow(P \otimes V) \otimes \mathbb{C}^{t} \rightarrow 0 .
$$

Since $L$ and $V$ are generic, we may assume for generic $P \in \Theta_{V}$ that $h^{0}(P \otimes L)=0$. As $\Theta_{V}$ is reduced, furthermore $h^{0}(P \otimes V)=1$ for generic $P \in \Theta_{V}$ by Theorem 1.1. Taking global sections, we obtain

$$
0 \rightarrow H^{0}(P \otimes E) \rightarrow H^{0}(P \otimes V) \otimes \mathbb{C}^{t} \rightarrow H^{1}(P \otimes L) \rightarrow \cdots
$$

By Riemann-Roch, $h^{1}(P \otimes L)=1$. By exactness, $h^{0}(P \otimes E) \geq t-1$ for all $P \in \Theta_{V}$. Since $E \subset W$, we have $h^{0}(P \otimes W) \geq t-1$ for all $P \in \Theta_{V}$. Thus by Theorem[1.1, the theta divisor $\Theta_{W}$, if defined, has multiplicity at least $t-1$ at all $P \in \Theta_{V}$, and thus is of the form $(t-1) \Theta_{V}+R_{W}$ where $R_{W}$ is a divisor on $J$ algebraically equivalent to $(n+1+m) \Theta$.

Now we check that the theta divisor of a generic such $W$ is defined. Since $L$ is generic of degree -1 , we have $h^{0}(N \otimes L)=0$ for all $N \in J$ outside a locus of codimension at least 2. Therefore, for generic $N \in J \backslash \operatorname{Supp} \Theta_{V}$, any map $N^{-1} \rightarrow W$ must lift from a map $N^{-1} \rightarrow M$. By Hirschowitz's Lemma [15, Theorem 1.2] and Riemann-Roch, we have moreover $h^{0}(N \otimes M)=1$.

As $N^{-1}$ is a subsheaf of $M$, the induced map $H^{1}(\operatorname{Hom}(M, E)) \rightarrow H^{1}\left(\operatorname{Hom}\left(N^{-1}, E\right)\right)$ is surjective. Since the latter space is nonzero, the map $N^{-1} \rightarrow M$ does not lift to a generic extension $0 \rightarrow E \rightarrow W \rightarrow M \rightarrow 0$. In particular, a generic such $W$ has a well-defined theta divisor.

As for reducibility: it is easy to find an extension $W$ satisfying $h^{0}(P \otimes W)>0$ for at least one $P$ with $h^{0}(P \otimes E)=0$. For such a $W$ the divisor $\Theta_{W}$ also has a component containing $P$, hence distinct from $\Theta_{V}$, and so is reducible.

In particular, we have:

Corollary 3.4. (1) Let $X$ be a curve of genus $g \geq 5$. There exist stable bundles of rank $r$ over $X$ for all $r \geq 4$ (resp., $\geq 5$ ) with reducible (resp., reducible and nonreduced) theta divisors.

(2) If $g=4$ then there exist stable bundles of rank $r$ over $X$ for all $r \geq 4$ (resp., $r \geq 8$ ) with reducible (resp., reducible and nonreduced) theta divisors.

Proof. If $g \geq 5$ then we may set $n=1$ and $t=2$ or 3 and $m$ arbitrary. This gives (1).

As for (2): The only place above where we use the fact that $g \neq 4$ is to ensure that we can choose $t$ with $3 \leq t<n(g-1)$ when $n=1$. Thus if $g=4$, we can find stable bundles of rank $\geq 4$ with reducible theta divisors as in case (1).

If we set $n=2$ and $t=3$ and $m$ arbitrary, then the construction gives stable bundles of rank $\geq 8$ with reducible and nonreduced theta divisors. 
3.1. The residual divisor $R_{W}$. Consider a generic $W$ with extension class $\delta(W)$ and theta divisor $(t-1) \Theta_{V}+R_{W}$. As in [9, $\left.\S 5\right]$, we can give a geometric description of $R_{W}$ as follows:

Let $N$ be a generic line bundle of degree $g-1$ satisfying $h^{0}(N \otimes E)=0$ and $h^{0}(N \otimes M)=1$. Then by Riemann-Roch,

$$
\operatorname{Im}\left(m_{N}: H^{0}\left(K_{X} N^{-1} \otimes E^{*}\right) \otimes H^{0}(N \otimes M) \rightarrow H^{0}\left(K_{X} \otimes E^{*} \otimes M\right)\right)
$$

is of dimension 1. The association $N \mapsto \operatorname{Im} m_{N}$ defines a rational map

$$
\mu: J \rightarrow \mathbb{P} H^{0}\left(K_{X} \otimes E^{*} \otimes M\right) \cong \mathbb{P} H^{1}\left(M^{*} \otimes E\right)^{*} .
$$

Since $m_{N}$ is injective, $N$ belongs to the indeterminacy locus of $\mu$ if and only if $h^{0}(N \otimes E)>0$ or $h^{0}(N \otimes M)>1$.

We write $R_{W}^{\prime}$ for the complement of the indeterminacy locus in $R_{W}$, and $H_{W}$ for the hyperplane defined by $\delta(W)$ on $\mathbb{P} H^{0}\left(K_{X} \otimes E^{*} \otimes M\right)$.

Lemma 3.5. The set-theoretic intersection of $H_{W}$ and $\mu(J)$ is exactly $R_{W}^{\prime}$.

Proof. Suppose $N \in J$ lies outside the indeterminacy locus of $\mu$. We have

$$
0 \rightarrow H^{0}(N \otimes W) \rightarrow H^{0}(N \otimes M) \stackrel{\cdot \cup \delta(W)}{\longrightarrow} H^{1}(N \otimes E) \rightarrow \cdots
$$

Then $N \in R_{W}$ if and only if the (up to scalar) unique section $\sigma$ of $N \otimes M$ satisfies $\sigma \cup \delta(W)=0$. By hypothesis, $h^{0}(N \otimes M)=1=h^{1}(N \otimes E)$, so this is equivalent to

$$
\cdot \cup \delta(W)=0 \in \operatorname{Hom}\left(H^{0}(N \otimes M), H^{1}(N \otimes E)\right) .
$$

Now it is well known that via Serre duality, the cup product map

$$
H^{1}(\operatorname{Hom}(M, E)) \rightarrow \operatorname{Hom}\left(H^{0}(N \otimes M), H^{1}(N \otimes E)\right)
$$

is dual to $m_{N}$. Thus $h^{0}(N \otimes W)>0$ if and only if $m_{N}^{*} \delta(W)=0$; in other words, $\mu(N) \in H_{W}$. The lemma follows.

\section{Symplectic eXtensions And Liftings}

Recall that a vector bundle $W$ is symplectic if there is an antisymmetric isomorphism $W \stackrel{\sim}{\rightarrow} W^{*}$; equivalently, if there exists a global bilinear nondegenerate antisymmetric form on $W$. In this section we gather some facts about such bundles.

Criterion 4.1. Let $E \rightarrow X$ be a simple vector bundle and $0 \rightarrow E \rightarrow W \rightarrow E^{*} \rightarrow 0$ an extension of class $\delta(W) \in H^{1}\left(\operatorname{Hom}\left(E^{*}, E\right)\right)=H^{1}(E \otimes E)$. Then $W$ carries a symplectic form with respect to which $E$ is isotropic if and only if $\delta(W)$ belongs to the subspace $H^{1}\left(\mathrm{Sym}^{2} E\right)$.

Proof. This is a special case of [8, Criterion 2.1].

Proposition 4.2. Let $E$ be any vector bundle and $F \subseteq E$ a subbundle. Then

$$
(F \otimes E) \cap \mathrm{Sym}^{2} E=\mathrm{Sym}^{2} F .
$$


Proof. The question is local. For some $x \in X$, suppose

$$
\left.\left.\sum e_{i} \otimes f_{i} \in(E \otimes F)\right|_{x} \cap \operatorname{Sym}^{2} E\right|_{x}
$$

where each $\left.e_{i} \in E\right|_{x}$ and $\left.f_{i} \in F\right|_{x}$. Furthermore, we assume that the sum is of minimal length (equal to the rank of the associated map $\left.\left.E^{*}\right|_{x} \rightarrow F\right|_{x}$ ). Then

$$
\sum e_{i} \otimes f_{i}=\sum f_{i} \otimes e_{i}
$$

and so by minimality $e_{i}=f_{\rho(i)}$ for some permutation $\rho$ of the indices. Hence all the $e_{i}$ belong to $\left.F\right|_{x}$. The proposition follows.

Now let $E$ be a vector bundle and $F \subset E$ a subbundle, and write $G:=E / F$. If $h^{0}(G \otimes E)=0$, then using Proposition 4.2 we find a diagram

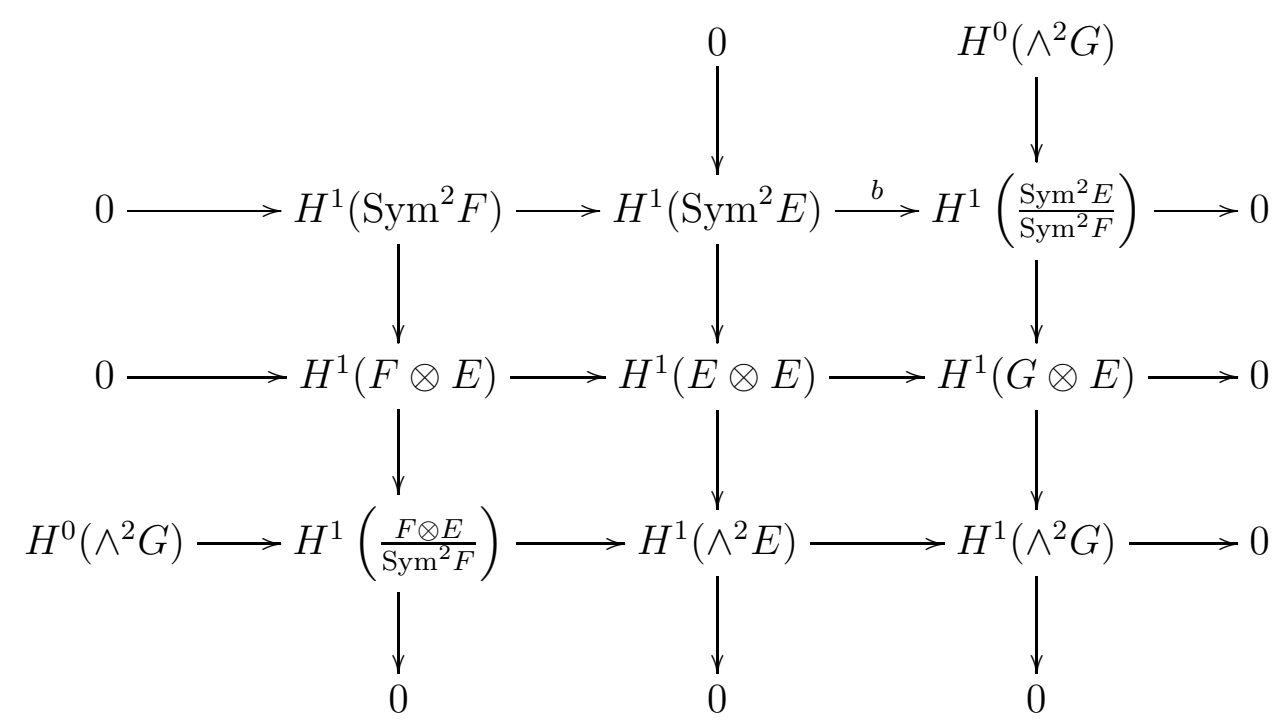

Lemma 4.3. Let $E, F$ and $G$ be as above. Then the subbundle $G^{*} \subseteq E^{*}$ lifts to $W$ if and only if $\delta(W)$ belongs to the preimage of $H^{0}\left(\wedge^{2} G\right)$ via the map $b$ in (4.1). In particular, if $h^{0}\left(\wedge^{2} G\right)=0$ then $G^{*}$ lifts to $W$ if and only if $\delta(W) \in H^{1}\left(\operatorname{Sym}^{2} F\right)$.

Proof. It is well known that $G^{*}$ lifts to $W$ if and only if $\delta(W)$ belongs to

$$
\operatorname{Ker}\left(H^{1}(E \otimes E) \rightarrow H^{1}(G \otimes E)\right)=H^{1}(F \otimes E) .
$$

Thus we must describe $H^{1}(F \otimes E) \cap H^{1}\left(\operatorname{Sym}^{2} E\right)$. In (4.1), we have

$$
H^{1}(F \otimes E) \cap H^{1}\left(\operatorname{Sym}^{2} E\right)=\operatorname{Ker}\left(H^{1}\left(\operatorname{Sym}^{2} E\right) \rightarrow H^{1}(E \otimes E) \rightarrow H^{1}(G \otimes E)\right) .
$$

By commutativity, this coincides with

$$
\operatorname{Ker}\left(H^{1}\left(\operatorname{Sym}^{2} E\right) \stackrel{b}{\rightarrow} H^{1}\left(\frac{\operatorname{Sym}^{2} E}{\operatorname{Sym}^{2} F}\right) \rightarrow H^{1}(G \otimes E)\right) .
$$

Thus $H^{1}(F \otimes E) \cap H^{1}\left(\operatorname{Sym}^{2} E\right)$ is the preimage of $H^{0}\left(\wedge^{2} G\right)$ by $b$. The lemma follows. 


\section{SympleCtiC Bundles With ReduCIBle AND NONREDUCED THETA Divisors}

Here we adapt the construction of $\$ 3$ to produce stable symplectic bundles with reducible and nonreduced theta divisors. As before, suppose $X$ has genus $g \geq 4$. Let $L$ be a line bundle of degree -1 and $V$ a generic stable bundle of rank $n \geq 1$ and degree zero. Let $E$ be a generic extension $0 \rightarrow L \rightarrow E \rightarrow V \otimes \mathbb{C}^{t} \rightarrow 0$, where $t<n(g-1)$.

Lemma 5.1. A generic symplectic extension $0 \rightarrow E \rightarrow W \rightarrow E^{*} \rightarrow 0$ is a stable vector bundle.

Proof. From Lemma 2.2 it follows that every nonzero quotient of $E^{*}$ has positive degree, and hence every proper subbundle of $E^{*}$ has nonpositive degree. Since $W$ is nonsplit, it is semistable. Suppose $F \subset W$ is a subbundle of degree zero. Then we have a diagram

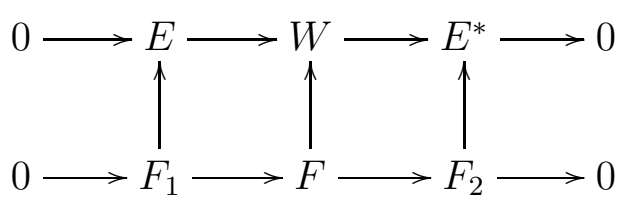

where $F_{1}$ is a subbundle of $E$ and $F_{2}$ a subsheaf of $E^{*}$. We distinguish three cases:

(i) If $F_{1} \neq 0$ then $\operatorname{deg} F_{1} \leq-1$ by Lemma 2.2 , whence $F_{2}=E^{*}$ and $\operatorname{deg} F_{1}=-1$. By Corollary 2.5 (1), the bundle $F_{1}$ is an extension $0 \rightarrow L \rightarrow F_{1} \rightarrow V \otimes \Lambda \rightarrow 0$ where $\Lambda$ is a (possibly zero-dimensional) subspace of $\mathbb{C}^{t}$.

(ii) If $F_{1}=0$ and $\operatorname{rk} F_{2}<\operatorname{rk} E$, then $F=F_{2}$ is a proper subbundle of degree zero. By dualizing the statement of Lemma 2.3, we see that $F$ is of the form $V^{*} \otimes \Pi$ for a nonzero subspace $\Pi \subset\left(\mathbb{C}^{t}\right)^{*}$.

(iii) If $F_{1}=0$ and $\operatorname{rk} F_{2}=\operatorname{rk} E$ then $F=F_{2}$ is an elementary transformation of $E^{*}$ along a torsion sheaf of length 1 .

We deal with each of these possibilities in turn:

(i) Here we obtain a diagram

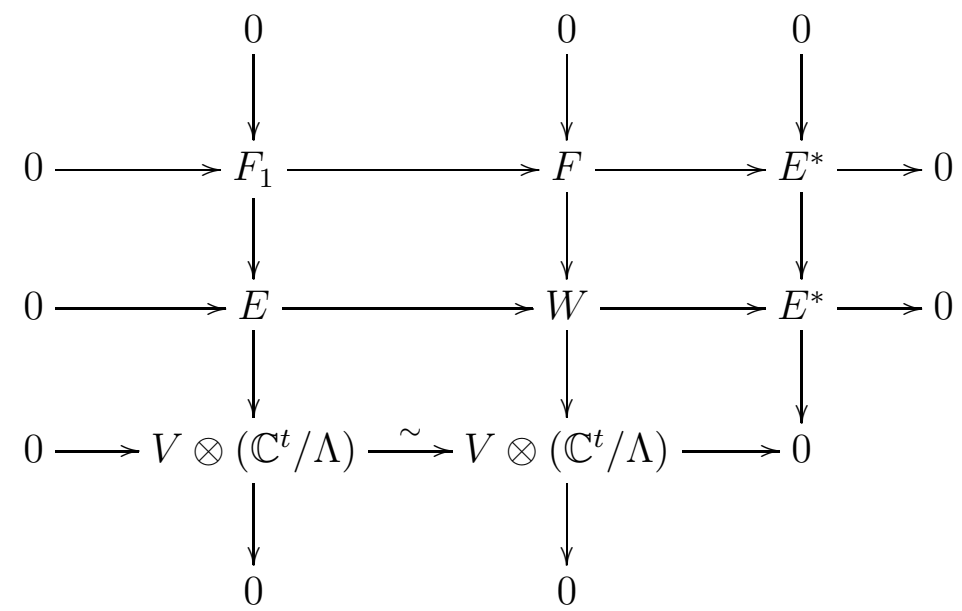


Dualizing this diagram, we see that $W^{*}$ contains a subbundle of the form $V^{*} \otimes\left(\mathbb{C}^{t} / \Lambda\right)^{*}$ lifting from $E^{*}$. But since $W$ is self-dual, this means that we are also in situation (ii). Thus it suffices to exclude possibility (ii) in general.

(ii) Clearly it suffices to treat the case $\operatorname{dim} \Pi=1$. We show that for any inclusion $j: V^{*} \otimes \Pi \rightarrow E^{*}$, we have

$\operatorname{dim} \operatorname{Ker}\left(j^{*}: H^{1}\left(\operatorname{Sym}^{2} E\right) \rightarrow H^{1}\left(\operatorname{Hom}\left(V^{*} \otimes \Pi, E\right)\right)\right)+\operatorname{dim} \mathbb{P}^{t-1}<h^{1}\left(\operatorname{Sym}^{2} E\right)$.

We write $F_{\Pi}$ for the subbundle of $E$ defined by the diagram

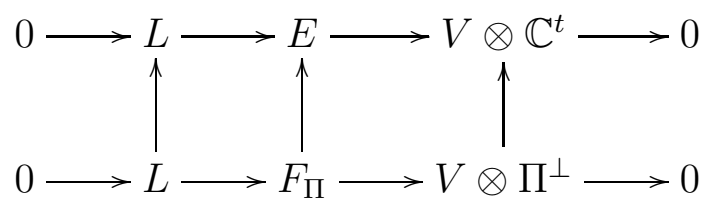

On the vector bundle level, clearly we have

$$
\operatorname{Ker}\left(j^{*}: \operatorname{Hom}\left(E^{*}, E\right) \rightarrow \operatorname{Hom}\left(V^{*} \otimes \Pi, E\right)\right) \cong \operatorname{Hom}\left(F_{\Pi}^{*}, E\right) .
$$

Hence by Proposition 4.2, we have $\operatorname{Ker}\left(j^{*}\right) \cap \mathrm{Sym}^{2} E=\operatorname{Sym}^{2} F_{\Pi}$. Furthermore, $h^{0}(V \otimes E)=0$ by Lemma 2.2. Therefore, by Lemma 4.3, to show that situation (ii) does not arise in general, it suffices to show that

$$
h^{1}\left(\operatorname{Sym}^{2} F_{\Pi}\right)+h^{0}\left(\wedge^{2}(V \otimes \Pi)\right)+t-1<h^{1}\left(\operatorname{Sym}^{2} E\right) .
$$

Since $V$ is generic, $h^{0}\left(\wedge^{2}\left(V \otimes \mathbb{C}^{t}\right)\right)=0$. Then the required inequality follows from a computation using Riemann-Roch and the fact that $h^{0}\left(\operatorname{Sym}^{2} F_{\Pi}\right)=0$.

(iii) By Lemma 2.1, it suffices to show that $\operatorname{dim} \psi\left(\Delta_{E \otimes E}\right)<h^{1}\left(\mathrm{Sym}^{2} E\right)-1$. This follows from

which is clear.

$$
2 t n+1<(t n+2)+\frac{(t n+1)(t n+2)}{2}(g-1)-1
$$

In summary, a general symplectic extension $0 \rightarrow E \rightarrow W \rightarrow E^{*} \rightarrow 0$ admits no subbundles of nonnegative degree.

We now describe the theta divisor of a generic such $W$.

Theorem 5.2. A general symplectic extension $0 \rightarrow E \rightarrow W \rightarrow E^{*} \rightarrow 0$ has a reducible theta divisor $\Theta_{W}$ which scheme-theoretically contains $(t-1)\left(\Theta_{V}+\Theta_{V^{*}}\right)$. In particular, if $t \geq 3$ then $\Theta_{W}$ is nonreduced.

Proof. Exactly as in the proof of Theorem 3.3. we see that the theta divisor of a generic such $W$, if it exists, scheme-theoretically contains $(t-1) \Theta_{V}$. By Serre duality (see also Beauville [4, §2]) we have $\iota^{*} \Theta_{W}=\Theta_{W}$, where $\iota$ is the involution of $J$ induced by $N \mapsto K_{X} N^{-1}$. In general, $\iota^{*} \Theta_{V}=\Theta_{V^{*}}$ by Serre duality. Since $V$ is generic of degree zero, we may assume that $\Theta_{V} \neq \Theta_{V^{*}}$. Then $\Theta_{W}$ scheme-theoretically contains $(t-1)\left(\Theta_{V}+\Theta_{V^{*}}\right)$.

We check that $\Theta_{W}$ is defined. Choose a generic line bundle $N \in J \backslash \operatorname{Supp}\left(\Theta_{V}+\Theta_{V^{*}}\right)$. By Riemann-Roch and genericity, $h^{0}\left(N \otimes E^{*}\right)=1$, and the corresponding map $j: N^{-1} \rightarrow E^{*}$ is a vector bundle injection. Dualizing, we obtain an exact sequence $0 \rightarrow F \rightarrow E \rightarrow N \rightarrow 0$, where $F:=\left(E^{*} / N^{-1}\right)^{*}$. Now we claim that

$$
h^{1}\left(\operatorname{Sym}^{2} E\right)-h^{1}\left(\operatorname{Sym}^{2} F\right)>0 .
$$


Since $\operatorname{Sym}^{2} F$ is a subbundle of $\operatorname{Sym}^{2} E$, it has no global sections. Then a computation with Riemann-Roch shows that the left hand side of (5.2) is equal to 1 (the expected value).

By Lemma 4.3 (with $G=N)$, we have $h^{0}(N \otimes W)=0$ for a generic symplectic extension $0 \rightarrow E \rightarrow W \rightarrow E^{*} \rightarrow 0$. Hence such a $W$ has a well-defined theta divisor $\Theta_{W}$. As in Theorem 3.3, we check that $\Theta_{W}$ in general has at least one component distinct from $\Theta_{V}$. This completes the proof of Theorem 5.2.

We summarize as follows:

Corollary 5.3. (1) Let $X$ be a curve of genus $g \geq 5$. Then there exist stable symplectic bundles of rank $2 k$ for all $k \geq 3$ (resp., $k \geq 4$ ) with reducible (resp., reducible and nonreduced) theta divisors.

(2) If $g=4$ then there exist stable symplectic bundles of rank $2 k$ for all $k \geq 3$ (resp., $k \geq 7$ ) with reducible (resp., reducible and nonreduced) theta divisors.

Proof. If $g \geq 5$ then we may as before set $n=1$ and $t=2$ or 3 . This proves (1).

As for (2): Setting $n=1$ and $t=2$, we obtain stable symplectic bundles of rank 6 with reducible theta divisors as in case (1). If we set $n=2$ and $t=3$, we obtain stable symplectic bundles of rank 14 with reducible and nonreduced theta divisors.

5.1. The residual divisor. As in $\$ 3.1$, we may give a geometric description of the residual divisor $R_{W}$ defined by $\Theta_{W}=(t-1)\left(\Theta_{V}+\Theta_{V^{*}}\right)+R_{W}$. Replacing $M$ with $E^{*}$, we define a map

$$
\mu_{s}: J \rightarrow \mathbb{P} H^{0}\left(K_{X} \otimes \operatorname{Sym}^{2} E^{*}\right)
$$

by composing the projection $\mathbb{P} H^{0}\left(K_{X} \otimes E^{*} \otimes E^{*}\right)-\rightarrow \mathbb{P} H^{0}\left(K_{X} \otimes \operatorname{Sym}^{2} E^{*}\right)$ with $\mu$. Note that if $h^{0}\left(N \otimes E^{*}\right)=1=h^{0}\left(K_{X} N^{-1} \otimes E^{*}\right)$, then the image of

$$
m_{N}: H^{0}\left(N \otimes E^{*}\right) \otimes H^{0}\left(K_{X} N^{-1} \otimes E^{*}\right) \rightarrow H^{0}\left(K_{X} \otimes E^{*} \otimes E^{*}\right)
$$

intersects $H^{0}\left(K_{X} \otimes \wedge^{2} E^{*}\right)$ in zero, so the base locus of $\mu_{s}$ is no bigger than that of $\mu$.

As before, an extension $0 \rightarrow E \rightarrow W \rightarrow E^{*} \rightarrow 0$ defines a hyperplane $H_{W}$ on $H^{0}\left(K_{X} \otimes E^{*} \otimes E^{*}\right)$. Since $\delta(W)$ belongs to $H^{1}\left(\operatorname{Sym}^{2} E\right)$, this $H_{W}$ cuts out a hyperplane $H_{W}^{s}$ on $\mathbb{P} H^{0}\left(K_{X} \otimes \mathrm{Sym}^{2} E^{*}\right)$.

As before, we write $R_{W}^{\prime}$ for the complement of the base locus of $\mu_{s}$ in $R_{W}$.

Lemma 5.4. The set-theoretic intersection of $\mu_{s}(J)$ and $H_{W}^{s}$ is exactly $R_{W}^{\prime}$.

Proof. If $h^{0}\left(N \otimes E^{*}\right) \cdot h^{0}\left(K_{X} N^{-1} \otimes E^{*}\right)=1$, then exactly as in Lemma 3.5, we see that $\mu(N) \in H_{W}$ if and only if $h^{0}(N \otimes W)>0$. Write

$$
S: H^{0}\left(K_{X} \otimes E^{*} \otimes E^{*}\right) \rightarrow H^{0}\left(K_{X} \otimes \operatorname{Sym}^{2} E^{*}\right)
$$

for the projection. Since $\delta(W)$ is symmetric, $\delta(W) \circ S=\delta(W)$. Thus $\operatorname{Im} m_{N} \in$ $\operatorname{Ker} \delta(W)$ if and only if $\operatorname{Im}\left(m_{N} \circ S\right) \subseteq H_{W}^{s}$; in other words, $\mu_{s}(N) \in H_{W}^{s}$. The lemma follows.

Remark 5.5. Since $\Theta_{W}$ and $\Theta_{V}+\Theta_{V^{*}}$ belong to $|2(t n+1) \Theta|_{+}$and $|2 n \Theta|_{+}$respectively, clearly $R_{W}$ belongs to the subspace $|2(n+1) \Theta|_{+}$of $|2(n+1) \Theta|$. In fact $\mu_{s}$ is $\iota$-invariant by construction, and factorizes via the Kummer variety. 


\section{ApPendix A. RAYNAUd's EXAmple}

In this appendix we construct a stable rank 2 vector bundle over a bi-elliptic curve of genus $g \geq 3$ having a reducible theta divisor. This construction is attributed to M. Raynaud.

Let $X$ be a smooth projective curve of genus $g \geq 3$. We assume that $X$ is bi-elliptic, i.e., $X$ admits a degree 2 map to an elliptic curve $Z$

$$
\pi: X \rightarrow Z \text {. }
$$

We continue to write $J$ for the Picard variety of line bundles of degree $g-1$ over $X$, $H^{0}(V)$ for $H^{0}(X, V)$ and $h^{0}(V)$ for $\operatorname{dim} H^{0}(X, V)$.

Let $i$ be the sheet involution of $X$. The canonical bundle $K_{X}$ of $X$ equals $O_{X}(R)$, where $R$ is the ramification divisor of $\pi$. Let $M$ be a line bundle of degree $g$ on $Z$, and denote $L$ the degree 2 line bundle over $X$ defined by $K_{X} L=\pi^{*} M$. We choose a square $\operatorname{root} \zeta$ of $L$, so $\zeta^{2}=L$, and $\operatorname{deg} \zeta=1$. By Riemann-Roch, we have

$$
h^{0}(Z, M)=g \quad \text { and } \quad h^{0}\left(X, K_{X} L\right)=g+1 .
$$

Hence the image of the injective map

$$
\pi^{*}: H^{0}(Z, M) \hookrightarrow H^{0}\left(X, K_{X} L\right)
$$

determines a point $e \in\left|K_{X} L\right|^{*} \cong \mathbb{P}^{g}$. We obtain a commutative diagram

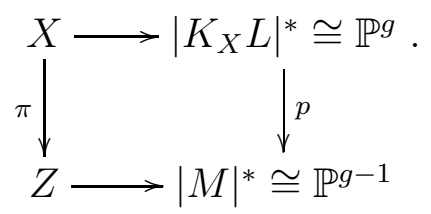

The map $p=\mathbb{P}\left(\pi^{*}\right)^{*}$ is projection with centre $e$. Moreover, the involution $i$ induces a decomposition into eigenspaces $H^{0}\left(\pi^{*} M\right)_{-} \oplus H^{0}\left(\pi^{*} M\right)_{+}$, where the second summand corresponds to $e$. We consider the rank 2 bundle $E$ with trivial determinant given by the extension class $e \in\left|K_{X} L\right|^{*}=\mathbb{P E x t}^{1}\left(\zeta, \zeta^{-1}\right)$. Hence $E$ fits into the exact sequence

$$
0 \rightarrow \zeta^{-1} \rightarrow E \rightarrow \zeta \rightarrow 0 \text {. }
$$

Proposition A.1. The bundle $E$ is stable.

Proof. The bundle $E$ is clearly semistable, since $e$ is nonzero. To see that is stable, it suffices by Lange-Narasimhan [11, Proposition 1.1] to show that $e$ does not belong to the image of $X$ in $\left|K_{X} L\right|^{*}$. Now the involution $i$ induces a decomposition into eigenspaces of $H^{0}\left(\pi^{*} M\right)_{-} \oplus H^{0}\left(\pi^{*} M\right)_{+}$, where the second summand defines the hyperplane $e \in H^{0}\left(K_{X} \zeta^{2}\right)^{*}$. If $H^{0}\left(\pi^{*} M\right)_{+}$is of the form $H^{0}\left(K_{X} L(-x)\right)$ for some $x \in X$ then the linear system $|M|$ on $Z$ would have the base point $\pi(x)$. But this is impossible since $\operatorname{deg} M>1$.

Given $z \in Z$, we denote by $\bar{z}$ the effective degree 2 divisor $\pi^{-1}(z)$. Note that for any $z \in Z$, the line spanned by $\bar{z}$ passes through $e$. By Lange-Narasimhan [11, Proposition 5.3], the set of line subbundles of maximal degree -1 of $E$ contains

$$
\left\{\zeta(-\bar{z}) \in \operatorname{Pic}^{-1}(X): z \in Z\right\} \cong Z \text {. }
$$


Let $\Theta_{E} \subset J$ be the theta divisor associated to $E$. We denote by $X^{d}$ and $X^{(d)}$ the $d$-th Cartesian and the $d$-th symmetric product of the curve $X$ respectively and by $q: X^{d} \rightarrow X^{(d)}$ the natural projection.

Lemma A.2. The natural map $\phi: Z \times X^{(g-2)} \rightarrow J$ defined by

$$
(z, D) \mapsto O_{X}(\bar{z}+D) \otimes \zeta^{-1}
$$

is birational onto its image, which is contained in the theta divisor $\Theta_{E}$. Therefore the divisor $\mathcal{D}_{1}:=\operatorname{Im} \phi$ is an irreducible component of $\Theta_{E}$.

Proof. Since we have injections $\zeta(-\bar{z}-D) \hookrightarrow \zeta(-\bar{z}) \hookrightarrow E$ for any pair $(z, D)$, we obtain an inclusion $\mathcal{D}_{1}=\phi\left(Z \times X^{(g-2)}\right) \subset \Theta_{E}$. Moreover, if the pair $(z, D)$ is general and $\phi(z, D)=\phi\left(z^{\prime}, D^{\prime}\right)$, then $z=z^{\prime}$ and $D=D^{\prime}$. In fact, the subvariety of line bundles $N \in \operatorname{Pic}^{g}(X)$ with $h^{0}(N) \geq 2$ is of dimension $g-2$. Since $\operatorname{dim} Z \times X^{(g-2)}=$ $g-1$, we obtain that $h^{0}\left(O_{X}(\bar{z}+D)\right)=1$ for a general $(z, D) \in Z \times X^{(g-2)}$.

For any integer $d$ the Norm map of the covering $\pi: X \rightarrow Z$ induces a morphism between the Picard varieties of degree $d$ line bundles

$$
\mathrm{Nm}: \operatorname{Pic}^{d}(X) \rightarrow \operatorname{Pic}^{d}(Z) .
$$

We recall the formula $N \otimes i^{*} N=\pi^{*} \operatorname{Nm}(N)$ for any line bundle $N \in \operatorname{Pic}(X)$. We write $P(X / Z)$ for the Prym variety of the cover $\pi: X \rightarrow Z$, i.e.,

$$
P(X / Z)=\text { Ker Nm }=\operatorname{Ker}\left(1+i^{*}\right)=\left\{\eta \in \operatorname{Pic}^{0}(X): i^{*} \eta=\eta^{-1}\right\} .
$$

Finally for any $\lambda \in \operatorname{Pic}(Z)$ the fiber $\operatorname{Nm}^{-1}(\lambda) \subset \operatorname{Pic}(X)$ is a translate of the Prym variety $P(X / Z)$. Note that $\operatorname{dim} P(X / Z)=g-1$ and that Ker Nm is connected since $\pi$ is ramified.

Lemma A.3. We put $\lambda=M \otimes \mathrm{Nm}(\zeta)^{-1} \in \mathrm{Pic}^{g-1}(Z)$ and $\mathcal{D}_{2}=\mathrm{Nm}^{-1}(\lambda) \subset J$. Then $\mathcal{D}_{2}$ is an irreducible component of the theta divisor $\Theta_{E}$. Moreover $\mathcal{D}_{2} \neq \mathcal{D}_{1}$.

Proof. We first show that $\mathcal{D}_{2} \subset \Theta_{E}$. The two subvarieties of line bundles $\eta \in$ $\operatorname{Pic}^{g-1}(X)$ satisfying $h^{0}\left(\zeta^{-1} \eta\right)>0$ and $h^{0}(\zeta \eta)>1$ respectively are of dimension $g-2$, so $h^{0}\left(\zeta^{-1} \eta\right)=0$ and $h^{0}(\zeta \eta)=1$ for a general line bundle $\eta \in \mathcal{D}_{2}$. Next, we observe that $\eta \in \mathcal{D}_{2}$ if and only if

$$
\eta \otimes i^{*} \eta=\pi^{*} \operatorname{Nm}(\eta)=\pi^{*} M \otimes \zeta^{-1} \otimes i^{*} \zeta^{-1}=K_{X} \otimes \zeta \otimes i^{*} \zeta^{-1},
$$

or equivalently

$$
i^{*}(\zeta \eta)=K_{X} \zeta \eta^{-1}
$$

We now tensor the exact sequence (A.1) with $\eta$ and take the associated long exact sequence of cohomology

$$
0 \longrightarrow H^{0}\left(\zeta^{-1} \eta\right) \longrightarrow H^{0}(E \eta) \longrightarrow H^{0}(\zeta \eta) \stackrel{\cup e}{\longrightarrow} H^{1}\left(\zeta^{-1} \eta\right) \longrightarrow \cdots
$$

Under the generality assumption for $\eta$ we have $h^{0}\left(\zeta^{-1} \eta\right)=0$ and $h^{0}(\zeta \eta)=1$, so $h^{0}(E \eta)>0$ if and only if the coboundary map $\cup e$ is zero, or equivalently, if the image of the multiplication map

$$
H^{0}(\zeta \eta) \otimes H^{0}\left(K_{X} \zeta \eta^{-1}\right) \longrightarrow H^{0}\left(K_{X} \zeta^{2}\right)=H^{0}\left(\pi^{*} M\right)
$$


lies in the invariant part $H^{0}\left(\pi^{*} M\right)_{+}$defining the extension class $e$. We see that this is the case if $i^{*}(\zeta \eta)=K_{X} \zeta \eta^{-1}$ and $h^{0}(\zeta \eta)=1$. Hence for a general $\eta \in \mathcal{D}_{2}$ we have $h^{0}(E \eta)>0$, which implies that $\mathcal{D}_{2} \subset \Theta_{E}$.

It is clear that the restriction of the Norm map to $\mathcal{D}_{1}$ is not constant, hence $\mathcal{D}_{2} \neq \mathcal{D}_{1}$.

In fact, there are no other components. This is shown in the following

Proposition A.4. We have a decomposition into irreducible components

$$
\Theta_{E}=\mathcal{D}_{1}+\mathcal{D}_{2}
$$

Proof. Let $\Theta \subset J$ denote the Riemann theta divisor. In order to show the equality it will be enough to show the equality of intersection numbers

$$
\mathcal{D}_{1} \cdot \Theta^{g-1}+\mathcal{D}_{2} \cdot \Theta^{g-1}=\Theta_{E} \cdot \Theta^{g-1}=2 \Theta^{g}=2 g ! .
$$

First we compute $\mathcal{D}_{1} \cdot \Theta^{g-1}$. Let $\mathcal{L}=\phi^{*}\left(\mathcal{O}_{J}(\Theta)\right)$ denote the pull-back of the line bundle $\mathcal{O}_{J}(\Theta)$ to $Z \times X^{(g-2)}$. Since $\phi$ is birational by Lemma A.2, we have $\mathcal{D}_{1} \cdot \Theta^{g-1}=$ $\mathcal{L}^{g-1}$. We will use the following commutative diagram

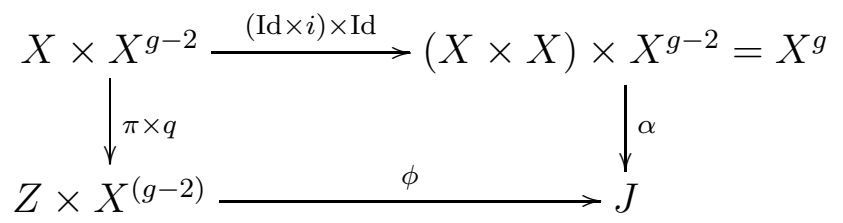

where $\alpha$ is given by $\alpha\left(\left(x_{i}\right)\right)=O_{X}\left(\sum_{i=1}^{g} x_{i}\right) \otimes \zeta^{-1}$. Hence, since $\operatorname{deg}(\pi \times q)=2(g-2)$ !, we obtain

$$
\mathcal{L}^{g-1}=\frac{1}{2(g-2) !}\left[(\pi \times q)^{*} \mathcal{L}\right]^{g-1}=\frac{1}{2(g-2) !}\left[(\operatorname{Id} \times i)^{*} \circ \alpha^{*} \mathcal{O}_{J}(\Theta)\right]^{g-1} .
$$

We will compute the latter intersection number in the cohomology $\operatorname{ring} H^{*}(X \times$ $\left.X^{g-2}, \mathbb{C}\right)$. A straightforward computation leads to

$$
\alpha^{*} \mathcal{O}_{J}(\Theta)=K_{X} \zeta \otimes K_{X} \zeta \otimes \cdots \otimes K_{X} \zeta\left(-\sum_{1 \leq i<j \leq g} \Delta_{i j}\right)
$$

where $\Delta_{i j} \subset X^{g}$ is the diagonal on the $i$-th and $j$-th component in $X^{g}$.

We need to recall some results from 13 . We denote by $\beta$ the generator of $H^{2}(X, \mathbb{Z}) \cong \mathbb{Z}$ induced by the orientation of $X$ and we choose generators $\alpha_{1}, \ldots, \alpha_{2 g}$ of $H^{1}(X, \mathbb{Z}) \cong \mathbb{Z}^{2 g}$ such that $\alpha_{j} \alpha_{k}=0$ unless $j-k= \pm g, \alpha_{j} \alpha_{g+j}=-\alpha_{g+j} \alpha_{j}=\beta$ for $1 \leq j \leq g$, and such that the involution $i$ acts as $i\left(\alpha_{1}\right)=\alpha_{1}, i\left(\alpha_{g+1}\right)=\alpha_{g+1}$ and $i\left(\alpha_{j}\right)=-\alpha_{j}, i\left(\alpha_{g+j}\right)=-\alpha_{g+j}$ for $2 \leq j \leq g$. We also introduce for $1 \leq k \leq g-2$ $\alpha_{j, k}=1 \otimes \cdots \otimes 1 \otimes \alpha_{j} \otimes 1 \otimes \cdots \otimes 1 \quad$ and $\quad \beta_{k}=1 \otimes \cdots \otimes 1 \otimes \beta \otimes 1 \otimes \cdots \otimes 1$, the $\alpha_{j}$ and $\beta$ being in the $k$-th place, as well as for $1 \leq j \leq 2 g$ $\xi_{j}=\alpha_{j, 1}+\cdots+\alpha_{j, g-2} \in H^{1}\left(X^{g-2}, \mathbb{C}\right) \quad$ and $\quad \eta=\beta_{1}+\cdots+\beta_{g-2} \in H^{2}\left(X^{g-2}, \mathbb{C}\right)$. We also put $\sigma_{i}=\xi_{i} \xi_{g+i}$ for $1 \leq i \leq g$ and we recall that we have (see [13]) the following relations $\sigma_{i}^{2}=0, \sigma_{i} \sigma_{j}=\eta^{2}$ for $i \neq j, \sigma_{i} \eta=\eta^{2}$, and $\xi_{i} \sigma_{j}=\sigma_{j} \xi_{i}$ for any $i, j$. 
We introduce the "diagonal" divisors in $X^{g-2}$ and in $X \times X^{g-2}$ with their reduced structures

$$
\begin{aligned}
& \Delta=\left\{\left(x_{1}, \ldots, x_{g-2}\right) \in X^{g-2} \mid x_{j}=x_{k} \text { for some } 1 \leq j, k \leq g-2\right\}, \\
& \Delta_{k}^{\prime}=\left\{\left(u ; x_{1}, \ldots, x_{g-2}\right) \in X \times X^{g-2} \mid u=x_{k}\right\} \text { and } \Delta^{\prime}=\sum_{k=1}^{g-2} \Delta_{k}^{\prime} .
\end{aligned}
$$

We denote by $p$ and $q$ the projection of $X \times X^{g-2}$ onto the first and second factor respectively. Then it follows from (A.3) that

$$
(\operatorname{Id} \times i)^{*} \circ \alpha^{*} \mathcal{O}_{J}(\Theta)=p^{*}\left(K_{X} \zeta \otimes i^{*} \zeta\right) \otimes q^{*}\left(\left(K_{X} \zeta\right)^{\otimes g-2}(-\Delta)\right)\left(-\Delta^{\prime}-i\left(\Delta^{\prime}\right)\right) .
$$

We now compute the class $c$ of this line bundle in $H^{*}\left(X \times X^{g-2}, \mathbb{C}\right)=H^{*}(X, \mathbb{C}) \otimes$ $H^{*}\left(X^{g-2}, \mathbb{C}\right)$. By [13] formula $(15.4)$ the class $[D] \in H^{*}(X, \mathbb{C}) \otimes H^{*}(X, \mathbb{C})$ of the diagonal $D \subset X \times X$ equals

$$
\begin{aligned}
{[D] } & =(g+1)(\beta \otimes 1+1 \otimes \beta)-\sum_{j=1}^{g}\left(\alpha_{j} \otimes 1+1 \otimes \alpha_{j}\right)\left(\alpha_{g+j} \otimes 1+1 \otimes \alpha_{g+j}\right) \\
& =(\beta \otimes 1+1 \otimes \beta)-\sum_{j=1}^{g}\left(\alpha_{g+j} \otimes \alpha_{j}+\alpha_{j} \otimes \alpha_{g+j}\right) .
\end{aligned}
$$

Let $\pi_{k}: X^{g-2} \rightarrow X$ denote projection onto the $k$-th factor. Then $\Delta_{k}^{\prime}=\left(\operatorname{Id} \times \pi_{k}\right)^{-1}(D)$ and therefore its class $\left[\Delta_{k}^{\prime}\right] \in H^{*}(X, \mathbb{C}) \otimes H^{*}\left(X^{g-2}, \mathbb{C}\right)$ equals

$$
\left[\Delta_{k}^{\prime}\right]=\beta \otimes 1+1 \otimes \beta_{k}-\sum_{j=1}^{g}\left(\alpha_{g+j} \otimes \alpha_{j, k}+\alpha_{j} \otimes \alpha_{g+j, k}\right)
$$

Summing over $k=1, \ldots, g-2$ leads to

$$
\left[\Delta^{\prime}\right]=(g-2) \beta \otimes 1+1 \otimes \eta-\sum_{j=1}^{g}\left(\alpha_{g+j} \otimes \xi_{j}+\alpha_{j} \otimes \xi_{g+j}\right)
$$

and, applying the involution $i$ on the first factor $X$

$$
\left[i\left(\Delta^{\prime}\right)\right]=(g-2) \beta \otimes 1+1 \otimes \eta-\alpha_{g+1} \otimes \xi_{1}-\alpha_{1} \otimes \xi_{g+1}+\sum_{j=2}^{g}\left(\alpha_{g+j} \otimes \xi_{j}+\alpha_{j} \otimes \xi_{g+j}\right) .
$$

Moreover, again by [13] formula (15.4), we have

$$
[\Delta]=(2 g-3) \eta-\sum_{j=1}^{g-2} \sigma_{j} \in H^{*}\left(X^{g-2}, \mathbb{C}\right),
$$

$\left[K_{X} \zeta \otimes i^{*} \zeta\right]=(2 g) \beta \in H^{2}(X, \mathbb{C})$, and $\left[\left(K_{X} \zeta\right)^{\otimes g-2}\right]=(2 g-1) \eta \in H^{2}\left(X^{g-2}, \mathbb{C}\right)$. Hence, using the preceding equalities, we can compute the class

$$
c=4(\beta \otimes 1)+2\left(\alpha_{g+1} \otimes \xi_{1}+\alpha_{1} \otimes \xi_{g+1}\right)+\sum_{j=1}^{g} 1 \otimes \sigma_{j} .
$$

We put $a=4(\beta \otimes 1)+2\left(\alpha_{g+1} \otimes \xi_{1}+\alpha_{1} \otimes \xi_{g+1}\right)$ and $b=\sum_{j=1}^{g} 1 \otimes \sigma_{j}$ and we note that $a b=b a$. Moreover, for dimensional reasons, $b^{g-1}=0$ and $a^{n}=0$ for $n \geq 3$. Hence 
$c^{g-1}=(a+b)^{g-1}=(g-1) a b^{g-2}+\frac{(g-1)(g-2)}{2} a^{2} b^{g-3}$. Using the above relations satisfied by the $\sigma_{i}$, we easily compute that $a b^{g-2}=4 \beta \otimes\left(\sum_{j=1}^{g} \sigma_{j}\right)^{g-2}=2 g ! \beta \otimes \eta^{g-2}, a^{2}=$ $-8 \beta \otimes \sigma_{1}$ and $a^{2} b^{g-3}=-4(g-1) ! \beta \otimes \eta^{g-2}$. Using the fact that $\beta \otimes \eta^{g-2}=(g-2) !$ under the canonical isomorphism $H^{2 g-2}\left(X^{g-1}, \mathbb{Z}\right)=\mathbb{Z}$, we obtain that $c^{g-1}=4((g-1) !)^{2}$. Hence $\mathcal{D}_{1} \cdot \Theta^{g-1}=\frac{c^{g-1}}{2(g-2) !}=2(g-1)(g-1) !$.

We now compute $\mathcal{D}_{2} . \Theta^{g-1}$. Let $L$ denote the restriction of the line bundle $\mathcal{O}_{J}(\Theta)$ to $\mathcal{D}_{2}$. We recall that $\mathcal{D}_{2}$ is a translate of the Prym variety $P(Y / X)$. Then by [5] Corollary 12.1 .5 the type of the polarization given by $L$ is $(1,1, \ldots, 1,2)$, hence $h^{0}\left(\mathcal{D}_{2}, L\right)=2=\frac{L^{g-1}}{(g-1) !}$ by the Riemann-Roch theorem applied to the Prym variety $P(X / Z) \cong \mathcal{D}_{2}$. Therefore $\mathcal{D}_{2} . \Theta^{g-1}=L^{g-1}=2(g-1)$ !.

We then conclude because we obtain equality (A.2) by summing both intersection numbers.

\section{REFERENCES}

[1] Ballico, E.; Russo, B.: Families of maximal subbundles of stable vector bundles on curves, Rocky Mt. J. Math. 31 (4) (2001), 1141-1150.

[2] Beauville, A.: Vector bundles on curves and generalized theta functions: recent results and open problems. "Current topics in complex algebraic geometry", 17-33, Math. Sci. Res. Inst. Publ. 28, Cambridge Univ. Press (1995).

[3] Beauville, A.: Some stable vector bundles with reducible theta divisor, Manuscripta Math. 110 (3) (2003), 343-349.

[4] Beauville, A.: Vector bundles and theta functions on curves of genus 2 and 3, Amer. J. of Math., 128 (3) (2006), 607-618.

[5] Birkenhake, C.; Lange, H.: Complex Abelian varieties, Grundlehren der mathematischen Wissenschaften, Vol. 302 (2003), Springer.

[6] Choe, I.; Hitching, G. H.: Secant varieties and Hirschowitz bound on vector bundles over a curve, Manuscripta Math. 133 (3-4) (2010), 465-477.

[7] Choe, I.; Hitching, G. H.: A stratification on the moduli spaces of symplectic and orthogonal vector bundles over a curve, arXiv:1204.0834, submitted.

[8] Hitching, G. H.: Subbundles of symplectic and orthogonal vector bundles over curves, Math. Nachr. 280, no. 13-14 (2007), 1510-1517.

[9] Hitching, G. H.: Rank four symplectic bundles without theta divisors over a curve of genus two, Internat. J. Math. 19 (2008), no. 4, 387-420.

[10] Hwang, J.-M.; Ramanan, S.: Hecke curves and Hitchin discriminant, Ann. Sci. Éc. Norm. Supér. (4) 37, no. 5, 801-817 (2004).

[11] Lange, H.; Narasimhan, M. S.: Maximal subbundles of rank two vector bundles on curves, Math. Ann. 266, no. 1 (1983), 55-72.

[12] Laszlo, Y.: Un théorème de Riemann pour les diviseurs thêta sur les espaces de modules de fibrés stables sur une courbe, Duke Math. J. 64, no. 2, 333-347 (1991).

[13] Macdonald, I.G.: Symmetric products of an algebraic curve, Topology 1 (1962) 319-343

[14] Raynaud, M.: Sections des fibrés vectoriels sur une courbe. Bull. Soc. Math. France 110 (1982), no. 1, pp. 103-125.

[15] Russo, B.; Teixidor i Bigas, M.: On a conjecture of Lange, J. Algebraic Geometry 8 (1999), 483-496. 
Høgskolen i Oslo og Akershus, Postboks 4, 0130 Oslo, Norway. Tel.: +47 2245 2146

E-mail address: george.hitching@hioa.no

Laboratoire J. A. Dieudonné, UMR CNRS 7351, Université de Nice Sophia-Antipolis, Parc Valrose, 06108 Nice Cedex 02, France. Tel.: +33 (0)4 92076202

E-mail address: pauly@math.unice.fr 